\title{
Frutas, legumes e verduras: recomendações técnicas versus constructos sociais
}

\author{
Fruits and vegetables: technical recommendations \\ versus social constructs
}

Fabio da Silva GOMES ${ }^{1,2}$

RE S U M O

Recomendações e prescrições de metas à população, muitas vezes, são construídas apenas com base em definições técnico-científicas, ignorando processos sócio-construtivos dos riscos que envolvem valores, percepções e experiências. Dessa forma, barreiras importantes podem dificultar o avanço de políticas de implementação dessas recomendações. Este artigo apresenta contribuições multidisciplinares à construção de recomendações e metas prescritas, essencialmente no que tange ao consumo de frutas, legumes e verduras, discutindo sobre algumas barreiras psicossociais e macro-estruturais para o consumo desses alimentos e suas implicações para as intervenções de base populacional. Assumindo um propósito reflexivo, o artigo conduz uma revisão crítica analisando a problemática em questão à luz de teorias estruturalistas sobre a construção social do risco. Recomendações e prescrições de metas definidas foram analisadas considerando o risco, seus determinantes sociais, componentes e conceitos agregados como fatores multidimensionais. Lições importantes extraídas da revisão incluem: 1) a necessidade de incorporar as contribuições populares à definição da agenda, conteúdo, estratégias de comunicação e implementação da política de alimentação; 2) a essencialidade da retomada dos aspectos não-nutricionais do alimento como componentes indispensáveis à valorização e promoção do consumo de frutas, legumes e verduras; e 3) a necessidade de adotar um conceito de alimentação saudável, que acompanhe a amplitude do conceito de saúde. A análise indica que as mensagens devem aproximar-se mais da valorização da cultura e tradição, evitando referências à alimentação saudável, essencialmente ou exclusivamente baseadas em nutrientes, doenças, longevidade e sofisticação.

Termos de indexação: cultura; programas e políticas de nutrição e alimentação; risco; sociologia; vegetais.

\section{A B S T R A C T}

Goal recommendations and prescriptions addressed to the population are often constructed based exclusively on technical-scientific definitions, ignoring social constructive processes of the risk that involves values, perceptions and experiences. Thus, important barriers may impair the advancement of policies that aim to

\footnotetext{
1 Ministério da Saúde, Instituto Nacional de Câncer, Coordenação de Prevenção e Vigilância, Área de Alimentação, Nutrição e Câncer. R. Dos Inválidos, 212, 4º andar, Centro, 20231-048, Rio de Janeiro, RJ, Brasil. E-mail: <fabiog@inca.gov.br>.

2 Instituto Brasileiro de Geografia e Estatística, Escola Nacional de Ciências Estatísticas. Rio de Janeiro, RJ, Brasil.
} 
670 | F.S. GOMES

implement these recommendations. This article presents multidisciplinary contributions to the construction of prescribed recommendations and goals, especially concerning the consumption of fruits and vegetables. It discusses some psychosocial and macro-structural barriers for the consumption of these foods and their implications for population-based interventions. With the objective of inducing reflection, the article conducts a critical review analyzing the problem under the light of structuralist theories on the social construction of risk. Recommendations and prescriptions of goals were analyzed considering the risk, its social determinants, components and concepts aggregated as multidimensional factors. Important lessons drawn from the review include: 1) the need to incorporate popular contributions to the definition, content, strategies of communication and implementation of the food policy agenda; 2) the vital need to recover the non-nutritional aspects of the foods, such as taste, as indispensable components to value and promote the consumption of fruits and vegetables; and 3) the need to adopt a concept of healthy eating that follows the broadness of the concept of health. The analysis indicates that the messages need to approach and value culture and tradition, avoiding references to healthy eating that are essentially or exclusively based on nutrients, diseases, longevity and sophistication.

Indexing terms: culture; nutrition programmes and policies; risk; sociology; vegetables.

\section{N T R O D U Ç Ã O}

O crescente aumento de problemas de saúde relacionados ao comportamento tem direcionado diversas instituições governamentais, não-governamentais, científicas e acadêmicas - de instâncias que vão desde o nível local até o internacional - ao desenvolvimento de ações de promoção de modos de vida mais saudáveis. A Estratégia Global para Alimentação, Atividade Física e Saúde, endossada pela Assembléia Mundial da Saúde 2004', pode ser descrita como a concretização ou formalização dessa preocupação no campo da nutrição e da atividade física.

De acordo com as evidências apresentadas pelo Relatório Mundial da Saúde 2003, a baixa ingestão de frutas, legumes e verduras (FLV) está entre os 10 principais fatores de risco que contribuem para mortalidade no mundo ${ }^{2}$. Estima-se que até 2,7 milhões de vidas poderiam ser salvas anualmente em todo o mundo, se o consumo de frutas, legumes e verduras fosse adequado ${ }^{3}$. As frutas, legumes e verduras, como parte da alimentação diária, poderiam ajudar a prevenir as principais doenças crônicas não-transmissíveis (DCNT) como as doenças cardiovasculares e os diversos tipos de câncer. Comer uma variedade de frutas, legumes e verduras garante, seguramente, uma adequada ingestão da maior parte dos micronutrientes, fibras e uma gama de fatores nutricionalmente essenciais. Além disso, o aumento do consumo de frutas, legumes e verduras pode ajudar a substituir alimentos que possuem altas concentrações de gorduras saturadas, açúcar e sal ${ }^{2-4}$.

Um relatório sobre alimentação, nutrição e prevenção de DCNT publicado pela World Health Organization/Food and Agricultural Organization of the United Nations (WHO/FAO), define, para a população, metas de ingestão de nutrientes e recomenda o consumo de, pelo menos, $400 \mathrm{~g}$ de frutas, legumes e verduras diariamente para a prevenção de DCNT, como doenças cardiovasculares, câncer, diabetes e obesidade 5 . O relatório afirma que há evidências convincentes de que as frutas, legumes e verduras diminuem o risco de obesidade e doenças cardiovasculares, e que as mesmas, provavelmente, diminuem o risco de diabetes. O relatório especifica que os tubérculos, como batatas e mandioca, não devem ser incluídos entre as frutas, legumes e verduras.

Uma revisão internacional, coordenada pela Agência Internacional para Pesquisa em Câncer - International Agency for Research on Cancer (IARC), concluiu que as frutas, legumes e verduras podem diminuir o risco de neoplasias malignas, particularmente as gastrintestinais. $O$ IARC estima que a fração prevenível da doença atribuída à baixa ingestão de frutas, legumes e verduras está entre $5 \%-12 \%$, podendo chegar a 20-30\% para cânceres das porções superiores do trato gastrintestinal, em todo o mundo ${ }^{6}$.

Essas evidências e recomendações têm sido transformadas em iniciativas de promoção 
do consumo de frutas, legumes e verduras, como os programas " 5 -ao-dia". Contudo, o aumento do consumo de frutas, legumes e verduras tem sido ainda muito limitado ${ }^{4}$, visto que barreiras remanescentes restringem e dificultam o alcance do que se deseja de fato - meta desejada -, resultando em exaustivos e, por vezes, infindos debates inter e intra-institucionais.

Os principais tópicos que envolvem o referido debate incluem: a meta desejada, seguida, especialmente, pelas barreiras governamentais, sociais, econômicas e culturais para atingir mudanças no comportamento alimentar da população. Esses obstáculos perpassam toda a estratégia de intervenção, da concepção à implementação. Por essa razão, não podem ser colocados de lado, nem ser descartados, devem ser enfrentados e gerenciados segundo as metas discutidas.

Este artigo promove uma abordagem ao debate analisando as metas delineadas e prescritas como derivações de riscos, os quais são freqüente e essencialmente, construídos por uma ótica unidimensional baseada no conhecimento científico, desconsiderando, muitas vezes, os determinantes e constituintes do risco e seus conceitos agregados - perigo, medo, confiança, proteção - como fatores multidimensionais.

Para tanto, o tema em questão será conduzido à luz da discussão sobre a construção social do risco e suas implicações para as intervenções voltadas a mudanças no comportamento alimentar populacional, em reflexão aos incessantes questionamentos como: Por que as pessoas não aderem aos apelos para mudança? O que distancia seu conhecimento de suas ações?

Esses questionamentos podem ser precedidos por questões como: As preocupações institucionais e populacionais são as mesmas? 0 que deve ser alvo de preocupação? Qual deve ser a meta desejada? Como são estabelecidas essas metas? A quem pertencem os desejos de atingir essas metas? Que riscos são elegíveis? Quais são eleitos?

\section{"Escolha" do risco}

A análise dos riscos pela sociedade é decorrente da apropriação reflexiva do conhecimento, o que significa que a aceitabilidade dos riscos é constantemente questionada pela sociedade. Porém, as análises da determinação desses riscos repousam inicialmente sobre a atribuição dos especialistas e suas instituições como definidores técnicos e quantitativos dos riscos e seus limites. Por conseguinte, tais riscos e limites podem ser questionados e/ou ignorados pela população, caso desconsiderem a mediação das experiências de vida relacionadas às causas dos danos e à magnitude das conseqüências dos riscos vivenciados?.

A abordagem técnico-científica estima o risco utilizando cálculos quantitativos, métodos estatísticos, portanto, em geral, se limita a essa estimação, à simples comunicação à população e à administração desses riscos a partir da definição de níveis seguros ou níveis aceitáveis de risco. No entanto, a passividade dos supostos leigos proposta por essa análise, torna-se o ponto de partida das avaliações do risco como um sistema social ${ }^{8}$ constituído de bases culturais sócio-construtivas ${ }^{9}$ indicando que os indivíduos são organizadores ativos de suas percepções, impondo seus próprios significados aos fenômenos ${ }^{10}$. Suas crenças e práticas desenvolvem uma liderança sobre o que identificam ${ }^{11,12}$ como risco $^{13}$.

Quando uma nova recomendação é anunciada, mesmo existindo credibilidade e confiança na instituição ou fonte de informação responsável pela prescrição, certa resistência em aceitá-la, no sentido de colocá-la em prática, ainda será experimentada, principalmente, quando essa prescrição requer ou exige uma mudança de comportamento que aflige valores e experiências anteriores. Por essa razão, a simples prescrição unilateral não tem valor para o leigo, se não obedece e acompanha uma construção sócio-cultural do risco que a envolve. Como ressalta Giddens", "reconhecer a existência de um risco ou limites de risco é aceitar não só a possibilidade de que as coisas 
podem dar errado, mas de que essa possibilidade não pode ser eliminada", fenômeno cuja lógica passa pelas experiências antecedentes, essencialmente as culturais.

Dessa forma, são eleitas confiáveis fontes de informação diversas. A maioria dos estudos apresenta baixas taxas de confiança em informações sobre riscos alimentares provenientes de fontes governamentais ${ }^{14-16}$. Um alto grau de confiança tem sido reportado em relação aos profissionais de saúde, organizações de consumidores e organizações ambientais, em contraste com uma tendência de baixa confiança em empresas privadas e na indústria de alimentos ${ }^{14-17}$. No entanto, esses sentimentos e relações diferem de acordo com as distintas dimensões que definem a confiança em uma fonte de informação, e influenciam a mudança de atitude pós-informação ${ }^{15}$. Dimensões psicológicas como a expertise e a honestidade, por exemplo, compõem alguns desses sentimentos e relações, de modo que fontes de informação repletas de expertise, mas consideradas desonestas, são menos persuasivas ${ }^{15}$.

Peters et al. ${ }^{18}$ afirmam que a confiança e a credibilidade são determinadas pelo conhecimento e expertise, transparência e honestidade, bem como pela preocupação e pelo cuidado. Segundo Renn \& Levine ${ }^{19}$, competência, objetividade, honestidade, consistência e fé, estruturam a confiança. Já Johnson ${ }^{20}$ aponta a competência (experiência e eficiência da fonte), o cuidado (transparência e honestidade, e se a fonte leva em consideração o interesse público) e os valores consensuais (observando o quanto a fonte compartilha os valores populares), como as explicações das razões que levam à confiança ou desconfiança.

A confiança e credibilidade, apesar das diferenciadas componentes de influência, são elementos chave à determinação e consolidação dos riscos, bem como dos mecanismos de proteção contra esses riscos. A sociedade não se protege de algo que não julga ser arriscado para o indivíduo e/ou coletividade, por mais que uma instituição demonstre todas as provas técnico- -científicas. Principalmente, porque em uma sociedade que se apropria do conhecimento reflexivo, a apresentação de um comportamento ou alimento, como risco em um instante inicial, e como inofensivo em seguida, não passa despercebida. As informações científicas passam por julgamentos constantes, feitos não só pela comunidade científica. Veríssimo ${ }^{21}$, em uma de suas crônicas, após a desvinculação do ovo como vilão do colesterol, solicita um ressarcimento à comunidade científica de todos os ovos que deixara de comer durante o período em que o ovo foi, de certa maneira, proibido como risco para o aumento do colesterol, e já previra a futura liberação da manteiga.

A natureza do risco também influencia a escolha dos riscos pelas sociedades. Riscos que assumem dimensões que superam limites geográficos e culturais, como a poluição, são mais destacados do que os riscos restritos ao âmbito individual. Isso porque a escolha dos riscos mais relevantes (os que devem ser considerados) pela sociedade é feita a partir da identificação de medos comuns que levam à constituição de valores comuns?.

Adicionalmente, a consolidação de riscos na sociedade não necessariamente conduz essa a uma proteção daqueles, mesmo que tenham sido socialmente construídos e que estejam conectados ao sentimento de perigo, ameaça. A proteção individual e/ou coletiva desses riscos está condicionada a motivações e necessidades que, muitas vezes, superam esse risco. Assim, por exemplo, a necessidade de se adequar ao padrão de beleza vigente, de se parecer com atriz da telenovela, muitas vezes supera os medos e condições de risco que envolvem uma cirurgia plástica; os riscos de desenvolvimento de câncer de pele pela exposição solar podem não superar a sedução do bronzeado; o risco da ingestão de alimentos densamente energéticos, que possuem quantidades excessivas de sódio, gorduras saturadas, açúcar e são pobres em micronutrientes essenciais, pode não superar a necessidade de viver uma determinada sensação. Transformações 
no comportamento humano têm sido experimentadas globalmente desde o século XVI, frutos do dito processo civilizador e de suas injunções e proibições, as quais modelam o indivíduo conforme o padrão da sociedade ${ }^{22}$.

De fato, as práticas sociais contêm dimensões corporais ${ }^{23}$. Halkier ${ }^{24}$, empregando o conceito de espaço contestado do corpo inspirado pela bio-política de Foucault ${ }^{25}$ e pelo processo civilizador de Elias ${ }^{22}$, destaca a influência da dimensão corporal sobre a forma com que os consumidores lidam com os riscos, identificando dois pólos espaciais: (1) o pólo do controle, relacionado à disciplina, ordem, necessidade e racionalidade; e (2) o do desejo, associado ao prazer, impulso e liberdade. Desses pólos derivaram-se os consumidores: (1) preocupado, o qual marginaliza o desejo a partir do controle; (2) irritado, caracterizado pela relação conflituosa entre o desejo e o controle; e (3) pragmático, o qual não reflete sobre os riscos, demonstrando indiferença ${ }^{24}$.

Adicionalmente, fenômenos promovidos pelos sistemas e subsistemas sociais da modernidade, como a sociedade da informação e a cultura do consumo, estão acima de qualquer esforço cognitivo-comportamental ou estratégia do campo da psicologia, direcionados à mudança de comportamento no plano individual. Nesse momento, o leigo assume um papel indispensável na desconstrução e reconstrução de paradigmas e modelos que não se baseiam somente nos medos sociais ou nas ameaças técnico-científicas.

\section{Promoção do consumo de FLV: o hiato entre o desejado e o desejo}

Aumentar o consumo de frutas, legumes e verduras tem sido um dos principais desafios da atualidade para a saúde pública, de acordo com Kraisid Tontisirin, Diretor da Divisão de Alimentação e Nutrição da $\mathrm{FAO}^{26}$. Segundo o mesmo, há uma série de razões, diferenciadas entre as populações, que levam ao não-consumo de frutas, legumes e verduras - preço, conveniência, sabor e estigma, para citar algumas ${ }^{26}$. A evidência científica tem demonstrado e sustentado o consumo de frutas, legumes e verduras como altamente recomendado e, cada vez mais, instituições nacionais de saúde e agricultura, representantes da indústria e de organizações internacionais, incluindo a FAO, têm trabalhado para acessar esses obstáculos e discutir formas de promover o consumo de frutas, legumes e verduras ao redor do mundo ${ }^{26}$.

Segundo os objetivos definidos pela Iniciativa de Promoção de Frutas e Hortaliças 2003 - WHO/FAO, parte integrante da Estratégia Global para Alimentação e Atividade Física ${ }^{1,2}$, é necessário (1) aumentar a conscientização geral acerca do papel das frutas e hortaliças na prevenção de DCNT; (2) aumentar seu consumo mediante ações essenciais nas áreas de saúde pública e agricultura; (3) incentivar e apoiar o desenvolvimento e a implantação de estratégias nacionais de promoção do consumo que sejam sustentáveis e envolvam todos os setores; e (4) apoiar pesquisas em áreas pertinentes e desenvolver os recursos humanos necessários para elaborar e implantar programas de promoção do consumo de frutas e hortaliças ${ }^{2}$. A iniciativa advoga pelo desenvolvimento e a implantação de programas de promoção do consumo de frutas, legumes e verduras que sejam sustentáveis, abrangentes e intersetoriais. As abordagens intersetoriais de iniciativas voltadas para alimentação, nutrição e saúde são apontadas como importantes estratégias para controlar e reduzir o aumento da obesidade, doenças cardiovasculares e câncer em todo o mundo4.

As recomendações de consumo de frutas, legumes e verduras ${ }^{3,27}$ têm sido traduzidas em campanhas nacionais de promoção da saúde, incluindo os programas "5-ao-dia" nos Estados Unidos, Reino Unido ${ }^{28,29} \mathrm{e}$ em iniciativas similares em outros países desenvolvidos. No entanto, Lock et al. ${ }^{4}$ apontam que essas iniciativas devem ser consideradas metas mínimas, e que uma ampla mudança em muitos setores é necessária, visto que o sucesso em aumentar o consumo de frutas, legumes e verduras tem sido muito limitado. Ressaltam a necessidade de enfatizar a promoção 
de políticas que tenham como alvo os determinantes do consumo desses alimentos, ao invés de, simplesmente, focar na mudança do comportamento individual ${ }^{4}$ desconsiderando, por exemplo, o con-texto estrutural da produção de alimentos ${ }^{13}$

O posicionamento científico quanto aos benefícios das frutas, legumes e verduras pode, portanto, ser interpretado como consensual, restando uma pauta de discussão acerca da tradução do científico por outros saberes. Com o intuito de refletir sobre o papel e a inteligibilidade de alguns desses conhecimentos envolvidos, alguns referenciais teóricos serão apresentados.

No que tange ao conhecimento científico, a descoberta inclui um processo programado e sistemático de uma busca por respostas de problemas até então insolúveis, ou por novas abordagens a problemas dados como resolvidos, segundo a ótica paradigmática vigente. Segundo Kuhn ${ }^{30}$, os cientistas trabalham em função desse paradigma, sem o qual não haveria como organizar a pesquisa e analisar os dados obtidos. As teorias propostas são concretizadas e reproduzidas quando se tornam modelos para novas descobertas, o conhecimento passa, portanto, por um processo cumulativo.

Nesse contexto, a pesquisa científica tenta descobrir regularidades que ampliem os conhecimentos num campo específico de estudo, e que tragam progresso à ciência. Esse progresso ocorre, geralmente, de forma interativa, cumulativa e gradual pelas abordagens sucessivas do problema em questão, no qual cada solução encontrada ocorre em função da que foi encontrada anteriormente. É um mecanismo dinâmico e contínuo que coloca em xeque teorias e explicações, confrontando-as com hipóteses antagônicas.

No que se refere ao conhecimento tradicional, a apropriação reflexiva do conhecimento, descrita por Giddens ${ }^{8}$, em uma determinada proporção, rompe com ele na sociedade, visto que existem descontinuidades entre a modernidade $e$ a tradição. Analisando alguns contrapontos, observa-se que a tradição, apesar de dinâmica, resiste à mudança, representando continuidades do passado, presente e futuro, enquanto a reflexividade da vida social moderna é caracterizada por constantes exames e reformulações das próprias práticas sociais 8 , o que implica em uma revisão, inclusive, das práticas tradicionais, que acabam, por vezes, deixando de ser tradicionais.

As sociedades pós-tradicionais têm como características marcantes os processos de abandono, desincorporação e problematização da tradição ${ }^{31}$. Para analisar a ruptura da tradição por essas sociedades, é preciso resgatar a tradição e suas características, para evitar conclusões equivocadas. O mais importante e, conseqüentemente, mais frisado entre os autores é a separação de hábitos e costumes de tradição. Para Giddens ${ }^{31}$ "a tradição está ligada ao ritual e tem suas conexões com a solidariedade social, mas não é a continuidade mecânica de preceitos que é aceita de modo inquestionável"; Boyer ${ }^{32}$ reafirma dizendo que "não há nenhuma conexão necessária entre repetição e coesão social, e o caráter repetitivo da tradição é algo que tem de ser explicado".

No entanto, a tradição, apesar de apresentar uma interpretação, a reproduz e a transmite por meio dos guardiões que detêm a verdade, interpretação que não deixa de ser mística e protege a tradição por meio dos rituais que a perpetuam. Uma proteção que se encontra intimamente ligada à manutenção da integridade e autenticidade como meio mais importante de sua definição como tradição ${ }^{31}$.

O rompimento com a tradição impôs mudanças na estrutura política e na vida social pós-tradicional, que distanciaram a rotina e aproximaram o vício desencadeado por sistemas sociais pós-tradicionais como a sociedade do consumo. No entanto, a "autoridade" não deixou de existir como formuladora de normas de vinculação ${ }^{31}$, houve apenas uma reordenação e reformulação da forma de determinação dessa; muitas normas passaram a ser impostas subliminarmente à vida social, os comportamentos passaram a ser construídos, induzidos, criando um mundo de fantasia que supera o real. 
Os guardiões foram substituídos por especialistas, porém as qualidades especiais desses podem ser aprendidas e apreendidas, em princípio, por qualquer um que se dedique a elas, portanto, segundo Weber ${ }^{33}$, por mais que o poder político se concentre nas mãos de chefes de governo muito bem assessorados por técnicos e especialistas, aqueles sempre dependerão dos sábios, dos mestres, visto que esses possuem um poder secular maior.

Outra forma de conhecimento envolvida refere-se ao saber popular, que, por sua vez, "é elaborado sobre a experiência concreta, a partir das suas vivências", as quais diferem da vivência de um profissional, que por seu turno oferece seu saber pensando "que o da população é insuficiente, e por esta razão, inferior, quando, na realidade, é apenas diferente" ${ }^{134}$.

Conflituosos embates referentes à definição de metas e prescrições preventivas por profissionais da área de saúde pública têm como ponto de partida, de um lado, a pressuposição da "previsão" por parte dos profissionais, como eixo estruturante das estratégias de prevenção a qual per si implica no olhar para o futuro. Do outro, pessoas que vivenciam e/ou vivenciaram situações extremamente adversas, que conduzem suas vidas por meio da "provisão" como categoria principal, o que as remete a lembranças da fome e a outras dificuldades de sobrevivência, ocasionando uma focalização do olhar principal no passado, preocupado em prover o presente dia ${ }^{34}$.

Segundo Peregrino ${ }^{35}$, a hipótese popular "não separa a doença da dinâmica social em que ela ocorre. Encara-a como fenômeno social. Entende que está na melhoria da sua qualidade de vida, a 'cura' social para este mal". Isso pode ser claramente ilustrado pelo discurso de uma moradora de favela ${ }^{36}$, que apesar da insistência do médico em dizer que a tuberculose era causada por uma bactéria, descrevia-a como hereditária e com tratamento garantido pelo ar puro, descanso e boa alimentação.

Essa reflexão evidencia o longo percurso das recomendações desde a elaboração à concretização comportamental e consolidação social, indicando que, por mais que haja sustentação técnico-científica que comprove os riscos ou a proteção contra eles, o baixo consumo de frutas, legumes e verduras só passará a ser um risco, quando tiver passado por sua construção social.

No Brasil, a construção do risco do baixo consumo de frutas, legumes e verduras, e de qualquer outro que esteja relacionado à alimentação é, necessariamente, antecedida pelo estigma da fome, caracterizado por uma luta histórica, cercada por diferentes visões de determinação dessa, as quais, ao longo dos anos, delinearam diferentes conceitos cuja coexistência sugere, de certa forma, uma harmonia, ao se complementarem ${ }^{37}$. Em 1946, Josué de Castro, já prestava esclarecimentos sobre a trágica situação da fome no País, que não poderia mais ser atribuída a fenômenos naturais, mas a sistemas econômicos e sociais que poderiam ser transformados para o benefício da população ${ }^{38}$. No entanto, só na década de 1980 , em um marco histórico do conceito, é que o acesso aos alimentos passa a ser explicitado, e determinantes sócio-econômicos e as iniqüidades passam a ser discutidas. Em seguida, no final dessa década e início da década de 1990, a fome passa a compor um conceito mais abrangente de Segurança Alimentar e Nutricional ${ }^{39}$, o qual incorpora questões relativas não só à acessibilidade e à quantidade, mas também à saúde, à qualidade e à sustentabilidade, associadas ao direito de se alimentar.

Escolhas alimentares modernas são determinadas pela estrutura socioeconômica, e caracterizadas por: 1) "escassez de tempo para o preparo e consumo de alimentos"; 2) "presença de produtos gerados com novas técnicas de conservação e de preparo"; 3) "vasto leque de itens alimentares"; 4) "deslocamentos das refeições de casa para estabelecimentos que comercializam alimentos"; 5) "crescente oferta de preparações e utensílios transportáveis"; 6) "oferta de produtos provenientes de várias partes do mundo"; 7) "arsenal publicitário associado aos alimentos"; 8) 
"flexibilização de horários para comer agregada à diversidade de alimentos"; 9) "crescente individualização dos rituais alimentares" 40 .

Segundo Giddens", "os modos de vida produzidos pela modernidade nos desvencilharam de todos os tipos tradicionais de ordem social", apontando as mudanças ocorridas nos últimos três ou quatro séculos como as mais dramáticas e abrangentes entre a maioria dos períodos precedentes. As mudanças na vida social e na estrutura da sociedade contemporânea, impulsionadas por sistemas sociais modernos, afetaram muitos aspectos do dia-a-dia. Índices positivos correspondentes a comportamentos alimentares, de atividade física e de saúde, muitas vezes, têm sido substituídos por práticas e estilos de vida modernos, implicando em um arrefecimento cultural da sociedade.

Ao analisar a dimensão do acesso ao alimento sob um enfoque macroeconômico histórico-institucionalista, dotado de um referencial baseado na teoria francesa da regulação, é possível identificar que a arquitetura institucional da economia brasileira reflete o caráter estrutural da pobreza relativa e das desigualdades ${ }^{41}$.

Como resultado, os riscos assumidos em relação à alimentação ainda são essencialmente ligados à história do País, problemas relacionados aos excessos nutricionais, à má nutrição, ainda são pouco reconhecidos socialmente. A preocupação maior está mais centrada no comer, e não no que comer - privilegiando a provisão, discutida anteriormente.

\section{Barreira agrotóxica}

Os agrotóxicos têm sido apontados como uma importante barreira para o consumo de frutas, legumes e verduras, principalmente pela população, no entanto, as instituições técnico-científicas adotam um posicionamento pouco esclarecedor. Um estudo realizado em áreas metropolitanas da Espanha indicou que mais de $2 / 3$ da população estudada $(69,4 \%)$ considerava os agrotóxicos como fator de risco para o desenvolvimento de câncer ${ }^{42}$. Nos Estados Unidos diversos estudos demonstram uma grande preocupação dos consumidores com a segurança dos alimentos, particularmente relacionada aos resíduos de agrotóxicos ${ }^{43}$, e estudos conduzidos na América do Sul indicam que a percepção de risco dos Brasileiros é superada pela percepção de risco dos Chilenos, entretanto supera aquela encontrada nos Estados Unidos ${ }^{44,45}$.

A preocupação com a presença de agrotóxicos nos alimentos é tão antiga quanto a introdução desses químicos no controle de pragas e doenças que afetam a produção agrícola ${ }^{46}$. Uma imagem venenosa e danosa foi construída partindo do próprio fim ao qual se destinam os agrotóxicos.

A conquista da confiança na vigilância e, principalmente, no controle da aplicação desses produtos, requererá muitas evidências científicas; divulgações massivas voltadas ao esclarecimento de mitos e verdades acerca desse constructo social; e, indispensavelmente, melhorias nos mecanismos de regulação. Low et al. ${ }^{47}$, analisando o risco relacionado a resíduos de agrotóxicos em alimentos, sugeriram que a percepção de risco dos consumidores é mais acentuada do que justificada.

Em um primeiro passo, a diretoria de Alimentos e Toxicologia da Agência Nacional de Vigilância Sanitária (ANVISA) iniciou, em junho de 2001, o Programa de Análise de Resíduos de Agrotóxicos em Alimentos (PARA), com o objetivo de monitorar os níveis desses compostos em alimentos produzidos e vendidos no Brasil ${ }^{46}$. A iniciativa representou um grande avanço à fiscalização do uso de agrotóxicos no âmbito nacional. Nos últimos quatro anos têm sido monitorados os níveis residuais desses compostos em nove tipos de alimentos consumidos diariamente pela população brasileira: alface, banana, batata, cenoura, laranja, mamão, maçã, morango e tomate - escoIhidos em função do seu consumo anual per capita e da disponibilidade de métodos validados nos laboratórios que deveriam realizar as análises ${ }^{48}$.

O referido avanço torna-se evidente frente às considerações feitas pelo Coordenador Técnico 
do Programa a respeito da situação pregressa do País: "Nada mais deprimente do que assistir ao Brasil participar de reuniões do Codex Alimentarius sem dados laboratoriais sobre níveis de resíduos de agrotóxicos em seus alimentos, sem condições de argumentar com outros países quanto a questões, muitas vezes, de natureza essencialmente política envolvendo limites máximos de resíduos. Nada mais preocupante do que legislar quanto ao registro de novos agrotóxicos ou quanto à renovação de registros concedidos previamente, sem uma base de dados nacional que permita ao legislador um mínimo de segurança quanto ao efeito de suas decisões sobre a segurança alimentar da população" 48 . Tais afirmações refletem a debilidade de informações e a obscuridade com a qual se conduziam as discussões e decisões, com conseqüente vulnerabilidade perante a confiança social.

O referido avanço, no entanto, não exclui a complementariedade imprescindível do passo rumo ao controle e à regulação do uso de agrotóxicos, visto que esse passo não é contemplado pelos fins do Programa, e que, de acordo com os mais recentes resultados do PARA (2001-2004), agrotóxicos não recomendados para determinados alimentos têm sido utilizados indiscriminadamente ${ }^{46}$. O mais preocupante, portanto, passa a ser a debilidade do controle frente às informações e aos dados identificados.

\section{Medicalimento vs comida}

Em uma orquestra muito afinada, Cultura do Consumo e Sociedade da Informação vendem alimentos como experiências de sabor, deleite, afeto, recompensa, consolo, apelando para contribuições da vida social real, da hiper-realidade ${ }^{49,50}$ e, até, da tradição, para construção dessas mensagens.

No entanto, os profissionais de saúde, em especial os voltados à promoção da alimentação saudável, como contraponto, ainda insistem em promover uma seleção de alimentos vendendo nutrientes, proteção ou diminuição do risco de desenvolvimento de doenças. Por mais que se busque promover e valorizar o pluralismo cultural, a fonte de sua promoção se restringe ao poder antioxidante ou ao baixo índice glicêmico da cultura alimentar, em detrimento da tradição, dos rituais, do folclore que a envolvem.

\section{CONSIDERAÇÕ ES FIN A IS}

Mudanças que afligem o caráter sócio-cultural de forma muito incisiva, impactante e repentina, terão sua implementação dificultada, pois sofrerão uma grande resistência por parte da população, uma vez que a percepção de confiança e desconfiança em comunicadores de riscos alimentares apresenta extrema robuste $z^{15}$. Isso porque a adoção de um novo comportamento direcionado à redução da exposição a um risco e ao aumento da proteção contra outro, passa pela construção social desses riscos. Isto é, o risco passa por um julgamento social que decidirá se ele será eleito ou não um risco para uma determinada sociedade ou classe pertencente a essa.

As intervenções de base populacional devem ser baseadas em abordagens multidisciplinares, e devem compreender todas as esferas da vida social, das micro às macro estruturas sociais, econômicas e políticas. Além disso, assumir o discurso popular como uma interrupção necessária, mas sem conteúdo e valor ${ }^{34}$, significa negar culturas, singularidades, realidades. Significa forçar a população a uma ação ineficaz baseada em uma realidade ideal imaginária ${ }^{51}$. O diálogo e a interlocução entre o saber científico e o saber popular são indispensáveis à promoção da saúde, e devem moldar as estratégias de promoção de práticas alimentares saudáveis, como o estímulo ao consumo de frutas, legumes e verduras, enfatizando os argumentos não-nutricionais.

Apesar de grande parte das políticas de alimentação ser orientada pela concepção da "escolha informada", a representação de cidadãos ativos tem sido cada vez mais expressiva, apontando a necessidade de incorporação das contribuições populares à definição da agenda e 
conteúdo da política; e da comunicação e implementação dessa política ${ }^{52}$.

Os fenômenos descritos compõem um movimento inerente aos sistemas sociais instaurados nas sociedades modernas, os quais apresentam aspectos que, em geral, despertam grande resistência, em especial, por técnicos da área da saúde. No entanto, vale ressaltar que a aflição provocada pela fenomenologia apresentada não inclui a ausência ou impotência de esforços individuais desses atores, que são chaves transformadoras desse processo.

Um longo percurso ainda separa o risco estabelecido do reconhecido em muitos países, especialmente nos subdesenvolvidos e em desenvolvimento, no que diz respeito ao consumo de frutas, legumes e verduras. No entanto, a mudança do enfoque das abordagens técnico-científicas baseadas na imposição de medos à população, com a pretensão de controlar impulsos e emoções ${ }^{22}$ que comprometam a saúde, pode indicar um atalho via valorização da cultura, da tradição, distanciando a exclusividade das mensagens associadas a nutrientes, doença, longevidade, sofisticação.

A retomada do alimento como expressão cultural, como comida, como lembrança, como sabor, como prazer, é indispensável à valorização e à promoção do consumo de frutas, legumes e verduras, como componentes de uma alimentação saudável. O conceito de saúde já foi, há muito, ampliado, por essa razão uma alimentação promotora dessa saúde deve acompanhar a amplitude desse conceito, o qual é indissociável da realidade, perpassando as dinâmicas e os constructos sociais.

\section{A GRADECIMENTOS}

À Coordenação de Aperfeiçoamento de Pessoal em Ensino Superior, Ministério da Educação pelo suporte, e à Rosa W.D. Garcia, Inês R.R. Castro, Lavínia D.R. Pessanha e Sheila Rotenberg, pelo incentivo e apoio e pela riqueza de suas considerações.

\section{REFERÊ NCIAS}

1. World Health Organization. Resolution WHA57.17. Global strategy on diet, physical activity and health. Proceedings of the 57th World Health Assembly; 200422 May. Geneva: WHO; 2004.

2. World Health Organization. The World Health Report 2003. Shaping the future. Geneva: WHO; 2003.

3. World Health Organization. Diet, nutrition and the prevention of chronic diseases. Geneva: WHO; 1990.

4. Lock K, Pomerleau J, Causer L, Altmann DR, McKee $M$. The global burden of disease attributable to low consumption of fruit and vegetables: implications for the global strategy on diet. Bull World Health Organ. 2005; 83(2):100-8.

5. World Health Organization. Food and Agricultural Organization of the United Nations. Expert Report on Diet, Nutrition and the Prevention of Chronic Diseases. Geneva: World Health Organization/Food and Agricultural Organization of the United Nations; 2003. WHO Technical Report Series 916.

6. Vainio $H$, Bianchini F. IARC Handbooks of cancer prevention: fruit and vegetables. Lyon: IARC Press; 2003.

7. Renn O. Concepts of risk: a classification. In: Krimsky S, Golding D, editors. Social theories of risk. London: Praeger; 1992.

8. Giddens A. The Consequences of modernity. Cambridge: Polity Press; 1990.

9. Douglas M, Wildavsky A. Risk and culture. An essay on the selection of technical and environmental dangers. Berkeley: University of California Press; 1982.

10. Wildavsky A. Public policy. In: Davis B, editor. The genetic revolution: scientific prospects and public perceptions. Baltimore: The John Hopkins University Press; 1991.

11. Freire P. Pedagogia do oprimido. São Paulo: Paz e Terra; 1993.

12. Mezirow J. Fostering critical reflection in adulthood. San Francisco: Jossey-Bass Publishers; 1991.

13. Kriflik LS, Yeatman H. Food scares and sustainability: a consumer perspective. Health Risk Soc. 2005; 7(1):11-24.

14. Frewer LJ, Howard C, Hedderley D, Shepherd R. What determines trust in information about food related risks? Underlying psychological constructs. Risk Anal. 1996; 16(4):473-86.

15. Frewer LJ, Miles S. Temporal stability of the psychological determinants of trust: Implications 
for communication about food risks. Health Risk Soc. 2003; 5(3):259-71.

16. Smith AP, Young JA, Gibson J. Consumer information and BSE: credibility and edibility. Risk Decis Policy. 1997; 2(1):41-51.

17. Gutteling JM, Wiegman $\mathrm{O}$. The source of risk messages. In: Gutteling JM, Wiegman O, editors. Exploring risk communication. The Netherlands: Kluwer Academic Publishers; 1996.

18. Peters RG, Covello VT, McCallum DB. The determinants of trust and credibility in environmental risk communication: an empirical study. Risk Anal. 1997; 17(1):43-54.

19. Renn O, Levine D. Credibility and trust in risk communication. In: Kasperson RE, Stallen PJM, editors. Communicating risks to the public. The Netherlands: Kluwer Academic Publishers; 1991.

20. Johnson B. Exploring dimensionality in the origins of hazard-related trust. J Risk Res. 1999; 2(4): 325-54.

21. Veríssimo LF. A mesa voadora. Rio de Janeiro: Objetiva; 2001.

22. Elias N. O processo civilizador 1: uma história dos costumes. Rio de Janeiro: Jorge Zahar; 1994.

23. Frank A. For a sociology of the body: an analytical review. In: Featherstone M, Hepworth M, Turner BS, editors. The body social process and cultural theory. London: Sage; 1992.

24. Halkier B. Risk and food: environmental concerns and consumer practices. Int J Food Sci Tech. 2001; 36(5):801-12.

25. Foucault M. The History of Sexuality. Harmondsworth: Penguin Books; 1978.

26. Food and Agricultural Organization of the United Nations. Increasing fruit and vegetable consumption becomes a global priority [cited 2006 Mar 27]. Available from: http://www.fao.org/ english/newsroom/focus/2003/fruitveg1.htm

27. World Cancer Research Fund. American Institute for Cancer Research. Food, nutrition and the prevention of cancer: a global perspective. Washington (DC): American Institute for Cancer Research; 1997.

28. Centers for Disease Control and Prevention. National 5 A Day Partnership Plan 2001-2004. Atlanta: National Center for Chronic Disease Prevention and Health Promotion; 2002.

29. Department of Health. The NHS Plan: a plan for investment. A plan for reform. London: The Stationery Office; 2000.

30. Kuhn TS. A estrutura das revoluções científicas. 5a. ed. São Paulo: Perspectiva; 1993.
31. Giddens A. In Defence of sociology: essays, interpretations, and rejoinders. Cambridge: Polity Press; 1996.

32. Boyer P. Tradition as truth and communication. Cambridge: Cambridge University Press; 1990.

33. Weber M. Economy and society. Berkeley: University of California Press; 1978.

34. Valla VV. Procurando compreender a fala das classes populares. In: Valla VV, organizador. Saúde e Educação. Rio de Janeiro: DP\&A; 2000.

35. Peregrino M. Picada, beco, vielas: caminhos do saber [dissertação]. Niterói: Universidade Federal Fluminense; 1995.

36. Cunha MB. Parque proletário, grotão e outras moradas: saber e história nas favelas da Penha [dissertação]. Niterói: Universidade Federal Fluminense; 1995.

37. Gomes FS. Segurança alimentar e nutricional. In: Gomes FS, organizador. Culinária social. Rio de Janeiro: Instituto Victus; 2004.

38. Castro J. Geografia da fome. Rio de Janeiro: Cruzeiro; 1946.

39. Valente FLS. Do combate à fome à segurança alimentar e nutricional: o direito à alimentação adequada. In: Valente FLS, editor. Direito humano à alimentação: desafios e conquistas. São Paulo: Cortez; 2002.

40. Garcia RWD. Reflexos da globalização na cultura alimentar: considerações sobre as mudanças na alimentação urbana. Rev Nutr. 2003; 16(4): 483-92.

41. Bruno M. Crescimento econômico, mudanças estruturais e distribuição, as transformações do regime de acumulação no Brasil - uma análise regulacionista [tese]. Paris e Rio de Janeiro: École des Hautes Études en Sciences Sociales e Universidade Federal do Rio de Janeiro; 2002.

42. García M, Fernández E, Borràs JM, Nieto FJ, Schiaffino A, Peris $M$, et al. Cancer risk perceptions in an urban Mediterranean population. Int J Cancer. 2005; 117(1):132-6.

43. Williams PRD, Hammitt JK. Perceived risk of conventional and organic produce: pesticides, pathogens and natural toxins. Risk Anal. 2001; 21(2):319-30.

44. Nyland LG. Risk Perception in Brazil and Sweden. RHIZIKON: risk research report No. 15. Stockholm: Stockholm School of Economics; 1993.

45. Bronfman NC, Cifuentes LA. Risk perception in a developing country: the case of Chile. Risk Anal. 2003; 23(6):1271-85.

46. Brasil. Agência Nacional de Vigilância Sanitária. Diretoria de alimentos e toxicologia. Gerência geral 
de toxicologia. Controlando os agrotóxicos nos alimentos. Relatório de Atividades 2001-2004. Brasília: Anvisa; 2005.

47. Low F, Lin HM, Gerrard JA, Cressey PJ, Shaw IC. Ranking the risk of pesticide dietary intake. Pest Manag Sci. 2004; 60(9):842-8.

48. Nóbrega AW. Um esforço para garantir a segurança alimentar nacional: o programa de análise de resíduos de agrotóxicos - PARA. In: Peres F, Moreira JC, organizadores. É veneno ou é remédio: agrotóxicos, saúde e ambiente. Rio de Janeiro: Fiocruz; 2003.

49. Baudrillard J. Le Systéme des objets: la consommation des signes. Paris: Denoel/Gonthier; 1968.

50. Baudrillard J. Simulations. New York: Semiotext(e); 1983.
51. Gomes FS, Azeredo RR, Ramos RG, Gugelmin SA. Saucepan Revelry: Nourishing Citizenship. Proceedings of the 2 nd International Conference on Local and Regional Health Programmes; 2004 Oct; Canada, Québec; 2004. Abstract 165. Available from: http://archives.colloquequebec 2004.com/Detail.aspx?lang=EN\&actNo=308

52. Draper A, Green J. Food safety and consumers: constructions of choice and risk. Social Policy Adm. 2002; 36(6):610-25.

Recebido em: 26/4/2006

Versão final reapresentada em: 14/9/2007 Aprovado em: 9/10/2007 